\title{
Long Term Results of Bipolar Hemiarthroplasty in Treatment of Fracture Neck Femur
}

M.E.Abd El-Naby, E.M.Ibrahim, M.A.Meselhy and M.E.Mohammed

Orthopedic Surgery Dept., Faculty of Medicine, Benha Univ., Benha, Egypt

E-Mail:mohammed@gmail.com

\begin{abstract}
Femoral neck fractures are intracapsular fractures that occur in the proximal femur in the area beginning distal to articular surface of the femoral head and ending just proximal to the intertrochantric region. to evaluate the functional outcome of bipolar hemiarthroplasty in patients with intra capsular femoral neck fractures . and to determine the factors affecting the outcome and to see whether bipolar hemiarthroplasty meets desired end result or not; patient will be evaluated using Harris hip score and radiographic assessment. The present work was a retrospective study including twenty patients. These patients were be followed up for minimum five years postoperatively and traced to detect their actual hip function and performance. The early complicated patients would be excluded. The mean age of the patients was 73.4 years. The Harris hip score showed satisfactory results at the end of follow up three patients (15\%) had hip scores from 100 to 91 (excellent). eight patients (45\%) cases had hip scores 90 to 81 (good). Two patients (10\%) were rated 80 to 70 . (fair), and seven patients (35\%) were rated below 70(poor). The mean Harris hip score at the end of follow up was71.0 (range from 25.0- to97.0) with SD 22.4. It was found that the greater motion at the inner bearing correlated with better clinical results. Therefore the amount of motion at the two interfaces appears dependent on a number of variables and still a topic of debate.
\end{abstract}

Keywords: Bipolar, Hemiarthroplasty, Fracture Neck Femur.

\section{Introduction}

Femoral neck fractures constitute $53-60 \%$ of the hip fractures and two-thirds of these fractures are displaced. Femoral neck fractures occur most commonly in elderly females and are uncommon in patients under the age of 60 years [1].

A hip fracture is associated with increased mortality; half of the patients may die within 5 years. The treatment goals for femoral neck fractures are early return to a satisfactory functional status along with the minimization of mortality, morbidity and the need for re-operation [2].

Whether fractures of the femoral neck in elderly patients should be treated with internal fixation, hemiarthroplasty, or total hip replacement (THR) should be determined by the degree of fracture displacement, patient's age, functional demands, and risk profile such as level of cognitive function and degree of physical fitness [2].

While most authors advocate osteosynthesis for younger patients and for those with un-displaced fractures, there is still controversy as to how to treat displaced femoral neck fractures in elderly patients [3].

Internal fixation is therefore the method of choice in young patients with displaced intra-capsular fractures and in very frail elderly patients who are not medically fit for prosthesis surgery. Internal fixation has a high rate of non-union and is with inferior results compared to hemiarthroplasty [4].

However, for the relatively healthy, active, and mentally alert elderly patient, treatment is still controversy. In recent randomized, controlled trials total hip arthroplasties (THAs) have been shown to provide superior functional outcome to internal fixation as assessed by Harris hip score and European Quality of Life-5Dimensions [5].

Another study found that hemiarthroplasty (HA) provided a superior outcome than internal fixation as treatment for displaced fractures in the eldry. There appears to be a consensus that unipolar or bipolar hemiarthroplasty is the preferred treatment for displaced intracapsular fractures in elderly patients with low functional demands in the absence of Rheumatoid arthritis and osteoarthritis of the hip [6].

James Ennis Bateman and Gilberty in 1974 [7], introduced bipolar hemiarthroplasty, which is a selfarticulating prosthesis. Advantage of bipolar prosthesis is that erosion and protrusion of acetabulum would be less because, there is an dual articulation between the inner head and shell and acetabulum .

The fixed-head hemiarthroplasty is associated with high acetabular erosion and protrusion rates, which affect the clinical results and makes revision to a total hip arthroplasty difficult [8].

Reported advantages of hemiarthroplasty (HA) over total hip replacement (THR) are reduced dislocation rates, less complex surgery, shorter operation times, less blood loss, and lower initial costs [6].

These complications have led many surgeons to choose a bipolar design. The theoretical advantage of a bipolar hemiarthroplasty is to decrease acetabular erosion and wear and their associated symptoms. There seems, however, to be a growing opinion that treatment should be based on the patient's age, functional demands, and individual risk profile [7].

Opponents of the hemi arthroplasty are of the opinion that the chance of protrusion of the femoral 
head in the acetabulum and subsequently required revision operation in most cases favors the use of total hip replacement [8].

The aim of this theses is to evaluate the functional outcome of bipolar hemiarthroplasty in patients with intra capsular femoral neck fractures. And to determine the factors affecting the outcome and to see whether bipolar hemiarthroplasty meets desired end result or not; patient will be evaluated using Harris hip score and radiographic assessment.

\section{Patients and methods}

The present work was a retrospective study including twenty patients. These patients were be followed up for minimum five years post-operatively and traced to detect their actual hip function and performance. The early complicated patients would be excluded.

The ethical committee accepted the performance of the study and informed consents were taken from all the patients as regards performance of the study.

The patients were evaluated both clinical and radiologically pre-operative and post-operative, the functional outcome was evaluated by using of Harris hip score. all data were collected from hospital records an clinical and radiological evaluation at last visit was detected.

\subsection{Statistical analysis of the data}

Data were fed to the computer and analyzed using IBM SPSS software package version 20.0.Qualitative data were described using number and percent. Quantitative data were described using range (minimum and maximum) mean, standard deviation and median. Comparison between different groups regarding categorical variables was tested using Chisquare test. When more than $20 \%$ of the cells have expected count less than 5, correction for chi-square was conducted using Fisher's Exact test or Monte Carlo correction.

The distributions of quantitative variables were tested for normality using Kolmogorov-Smirnov test, Shapiro-Wilk test and D'Agstino test, also Histogram and QQ plot were used for vision test. If it reveals normal data distribution, parametric tests was applied. If the data were abnormally distributed, nonparametric tests were used. For normally distributed data, comparison between the two studied groups were done using independent t-test while for abnormally distributed data, comparison between the two groups were done using Mann Whitney test. Significance of the obtained results was judged at the $5 \%$ level.

\section{Results}

The mean age of the patients was 73.4 years , range from 66years old to 82 years old, the SD was $73.4 \pm 4.2$, Table (1)

Table (1) distribution of the studied group according to age.

\begin{tabular}{|c|c|c|c|}
\hline \multirow{2}{*}{ Age (years) } & & \multicolumn{2}{|c|}{$\operatorname{Bipolar}(n=20)$} \\
\hline & & No. & $\%$ \\
\hline $60-<70$ & & 3 & 15.0 \\
\hline $70-<80$ & & 15 & 75.0 \\
\hline$\geq 80$ & & 2 & 10.0 \\
\hline Min. - Max. & & \multirow{2}{*}{\multicolumn{2}{|c|}{$\begin{array}{c}66.0-82.0 \\
73\end{array}$}} \\
\hline Median & & & \\
\hline & Male & 8 & 40.0 \\
\hline Sex & Female & 12 & 60.0 \\
\hline
\end{tabular}

There were fifteen patient $9(75 \%)$ in whom the operation were done with lateral approach and fife patient through posterior approach. five patients $(25 \%)$ were operated with no need for blood transfusion, while the remaining fifteen patients
$(75 \%)$ received different amount of blood. The duration of surgery in this study ranged from $90 \mathrm{~min}$. up to $140 \mathrm{~min}$. with a mean value of $102.33 \pm 14.50$ minutes Table (2).

Tables (2) Distribution of the studied cases according to blood transfusion and surgical time.

\begin{tabular}{lcc}
\hline & No & $\%$ \\
\hline Blood transfusion & \multicolumn{2}{c}{25.0} \\
No & 5 & 75.0 \\
Yes & 15 & 60.0 \\
$\mathbf{1}$ unit & 9 & 20.0 \\
$\mathbf{2}$ units & 3 & 20.0 \\
$\mathbf{4}$ units & 3 & \multicolumn{2}{c}{$1.0-4.0$} \\
Min. - Max. & \multicolumn{2}{c}{$1.67 \pm 1.15$} \\
Mean \pm SD & \multicolumn{2}{c}{1.0} \\
Median & \multicolumn{3}{c}{} \\
Surgical time & \multicolumn{3}{c}{} \\
\hline
\end{tabular}




\begin{tabular}{lcc}
\hline Table (2) Continue & \\
\hline$\leq \mathbf{1 0 0}$ & 14 & 70.0 \\
$>\mathbf{1 0 0}$ & 6 & 30.0 \\
Min. - Max. & \multicolumn{2}{c}{$90.0-135.0$} \\
Mean \pm SD & $102.33 \pm 14.50$ \\
Median & \multicolumn{2}{c}{100.0} \\
\hline
\end{tabular}

The Harris hip score showed satisfactory results at the end of follow up three patients $(15 \%)$ had hip scores from 100 to 91 (excellent). eight patients (45\%) cases had hip scores90 to 81 (good). Two patients (10\%) were rated 80 to 70 . (fair), and seven patients $(35 \%)$ were rated below 70 (poor). The mean Harris hip score at the end of follow up was71.0 (range from 25.0- to97.0) with SD 22.4

At the end of follow up three patients treated with bipolar prosthesis (15\%) had no pain, seven patients $(35.0 \%)$ had slight pain, that did not compromise their activities, three patients $(15 \%)$ had mild pain not affecting their average activities, three patients $(15 \%)$ had moderate pain that caused some limitation of activity. three patients (15\%) had marked pain that caused serious limitation of activity. One patient was totally disabled, crippled and bed ridden.

Four patients could walk unlimited $(20 \%)$ two of them were males with mean age 63 and they were farmers and one of them returned to the pre injury level of activity. nine patients $(45 \%)$ could walk up to 600 meters, three $(15 \%)$ could walk up to 300 meters, four $(20 \%)$ could walk indoors only they were females with mean age of 69 years and two of them were complicated by infection and one was complicated by DVT. No one was bed ridden. The mean score for walking distance was 6.95(out of 11 points in the scoring system) Fig (1).

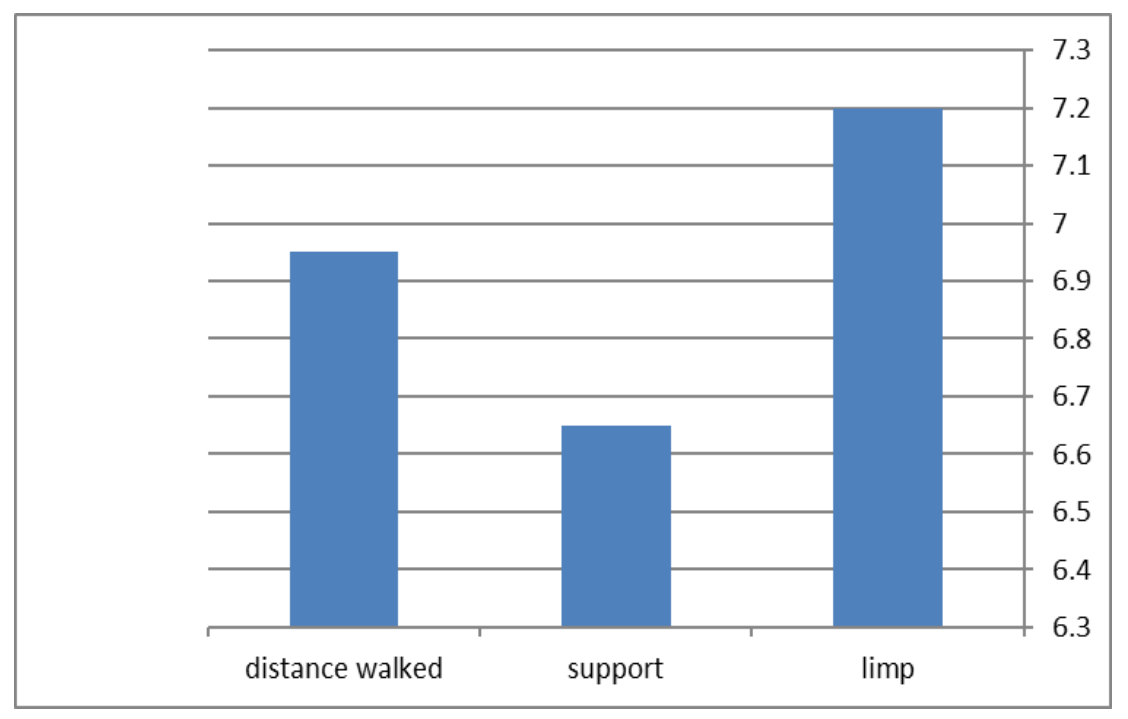

Fig (1) Chart showing gait score.

Stairs: ten patients treated with bipolar prosthesis $(50 \%)$ could climb the stairs normally, six patients $(30 \%)$ could climb the stairs using railing, two patients $(10 \%)$ could climb the stairs in any manner, and two patients $(10 \%)$ was unable to climb stairs. The mean score for climbing stairs in patients was 2.70 points (out of 4 points in the scoring system).

Tie shoes: fifteen patients treated with bipolar prosthesis $(75 \%)$ could tie shoes with ease, three patients (15\%) with difficulty, and two patients $(10 \%)$ was unable to tie shoes and socks. The mean score for Tie shoes was3.30 points (out of 4 points in the scoring system).
Sitting on chair: thirteen patients treated with bipolar prosthesis (65\%) were able to sit comfortably on a chair for one hour or more, and seven patients (35\%) were able to sit on a chair for less than one hour (high chair). No one was unable to sit comfortably on chair for 30 minutes. The mean score for sitting on chair in bipolar patient's was4.30 points (out of 5 points in the scoring system).

Use of transportation: fifteen $(75 \%)$ patients were able to use public transportation, and five patients $(25 \%)$ were unable to use public transportation Fige (2). 


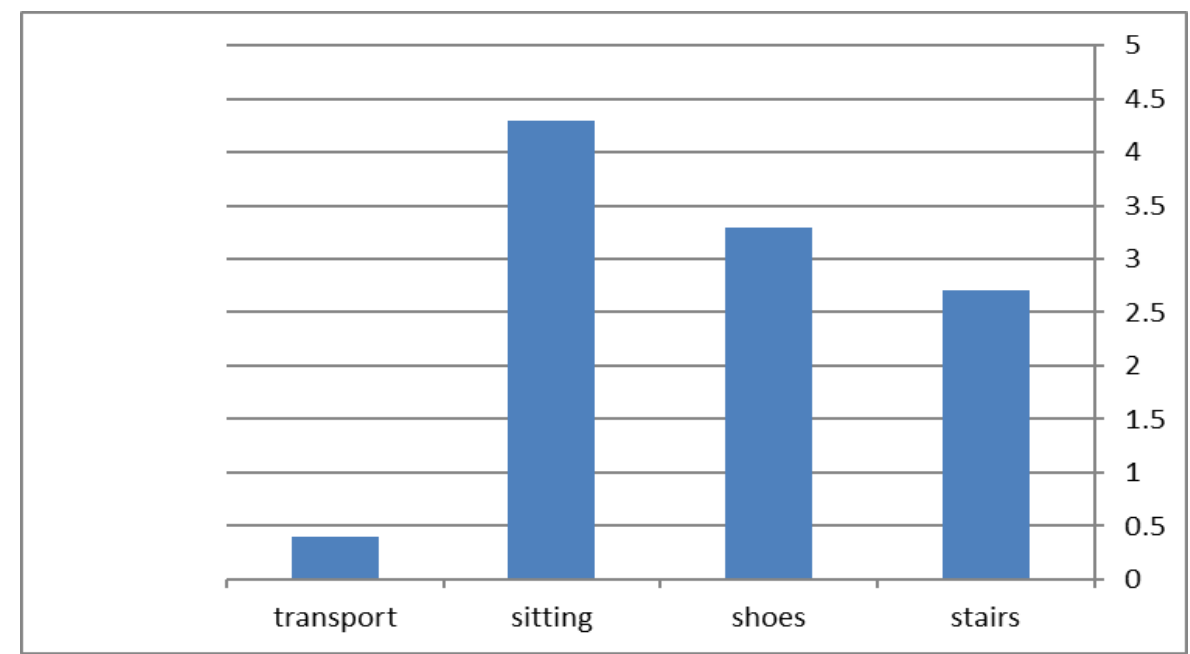

Fig (2) Chart showing activities score.

Radiographs of fractures ,contra lateral hip, postoperative, six month post operatively and then every year. were collected from patient records and the patient themselves. the radiological changes were compared with the native hip or contralateral hip. seven cases in this study sustained acetabular erosion which was grade 2 with narrowing of the joint space and migration of the prosthesis so the incidence of acetabular erosion in this study was $35 \%$. Fig (3).

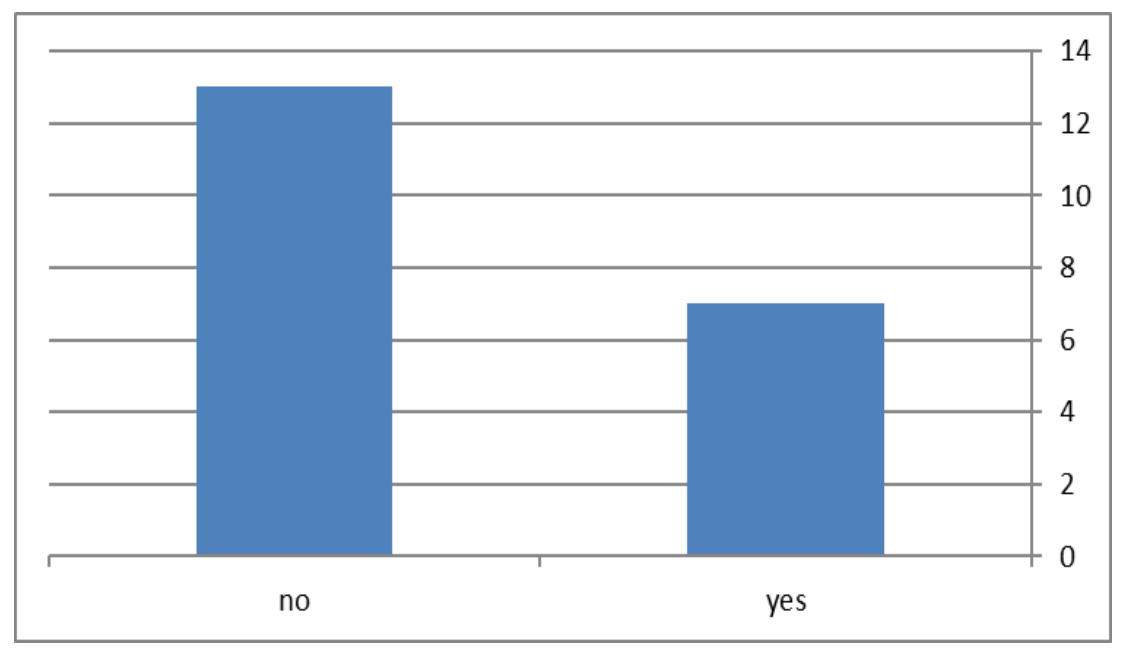

Fig (3) Chart showing incidence of acetabular erosion.

\section{Discussion}

The mean age in the present study was 73.4 years. The mean age of patients reported in Abdel Khalek $\mathrm{M}$ et al [9] is 78 years.

Philipp von roth etal [10] reported on twenty elderly patients treated by bipolar arthroplasty survived out of three handred fifty nine patient and the mean age was 79 years $(60 y-99 y)$.

Females were more affected than males in the present study with a ratio of 1.5-1.0.

Most studies reported higher incidence of the femoral neck fractures in elderly females and most probably this was due to the higher incidence of osteoporosis in females which makes females more prone to femoral neck fractures also the higher life expectancy of the females is another contributing factor [10].

In the present study the satisfactory results represented $55.0 \%$ of the cases and the mean final Harris Hip Score was 71.0 and the mean age was 73.4 years.

Haidukewych et al. studied 212 displaced intracapsular femoral neck fractures treated with bipolar hemiarthroplasty for a 20 year period and the satisfactory results represented about $83.2 \%$ of the cases with average Harris Hip Score of 86 [11].

Three patients $(15.0 \%)$ had no limping all of them had the femoral stem central and had negative Trendelenberg test and had no pain (pain score 44), twelve $(60.0 \%)$ had slight limping, three $(15 \%)$ had moderate limping, and two $(10 \%)$ had severe limping. 
One of them had deep infection and acetabular erosion, and one had muscle weakness and positive Trendelenburg test. The mean score for limping was 7.20 (out of 11 points in the scoring system).

Abdelkhalek M, and Abdelwahab M [9], in their study showed that $90 \%$ of their cases had no limping and $10 \%$ had slight limping. This result is better than the result in the present study and most probably the cause was that they performed the bipolar arthroplasty through posterior approach which spares the abductor muscles and allow early rapid rehabilitation

The percentage of patients who had no or slight limp in the study performed by Mazen $S$ et al [12], was $78.4 \%$ which is near to the results in the present study and they performed their cases through the trans gluteal lateral approach.

The cause of limping is attributed to muscle weakness, abductor insufficiency, pain, or leg length discrepancy.

In this study, five patients $(25.0 \%)$ could walk without support they all had no or slight limping, five $(25.0 \%)$ used a cane for long walks, six (30.0\%) used a cane most of the time, no one used one crutch, three (15\%) used two canes, and one (5.0\%) used two crutches. This patient was a female 69 year old who had deep infection and acetabular erosion with proximal and medial migration. The mean score for support was 6.65 (out of 11 points in the scoring system).

Mazen S, Julien G, and Riad F studied 51 femoral neck fractures treated by bipolar arthroplasty and showed that $51.4 \%$ of the patients could walk without support and $18.9 \%$ used cane for long walks and the age of the patients included in their study was higher reaching 77.6 years [12].

Kenzora et al [13], compared the results of bipolar arthroplasty with the results of unipolar arthroplasty in about 270 patients with displaced femoral neck fractures. The mean age was 65 years. They found that patients who received bipolar arthroplasty had less pain and better function with significantly faster walking speeds and less dependence on external walking support for a 2 year period compared to patients who received unipolar arthroplasty.

In this study, Four patients could walk unlimited $(20.0 \%)$ two of them were males with mean age 63 and they were farmers and one of them returned to the pre injury level of activity. nine patients $(45.0 \%)$ could walk up to 600 meters, three $(15.0 \%)$ could walk up to 300 meters, four $(20.0 \%)$ could walk indoors only they were females with mean age of 69 years and two of them were complicated by infection and one was complicated by DVT. No one was bed ridden. The mean score for walking distance was 6.95(out of 11 points in the scoring system).

Bangarshettar [14], found in his study that the majority $(84.4 \%)$ of the patients were able to walk for considerable distance and only $15.6 \%$ of patients were restricted to indoors.
Dixon S, and Bannister G [15], studied 35 active independent patients who were admitted with femoral neck fractures and were treated by bipolar arthroplasty for a period of 32 months and found that $84 \%$ of the patients could walk outdoors for considerable distance without restrictions.

N.R.Lestrange [16], studied hemiarthroplasty for femoral neck fractures in elderly and concluded that patients who had a bipolar hemiarthroplasty had better range of hip motion and faster walking speeds.

The walking ability of the patients are affected by the preinjury level of activity, the occurrence of complications, the early rehabilitation, the pain level, and the muscle power.

In this study, Ten patients $(50.0 \%)$ could climb the stairs normally, 6 patients(30.0\%) used railing, and four $(20.0 \%)$ were unable to climb stairs they were females one was complicated by early deep infection and acetabular erosion, and the other had DVT with painful movement.

S.Mazen [12], studied 51 femoral neck fractures treated by bipolar arthroplasty and found that $32.4 \%$ of the patients used stairs normally without railing and $45.9 \%$ used the railing.

In this study, Fifteen patients $(75.0 \%)$ could tie shoes with ease, three $(30.0 \%)$ with difficulty, and two $(10.0 \%)$ were unable to tie shoes.

This result was similar to the results of Gallinaro B et al [17], documented that $85 \%$ of his patients could tie shoes and socks.

In this study, Thirteen patients $(65.0 \%)$ were able to sit comfortably on a chair for one hour or more, seven $(35.0 \%)$ were able to sit on a chair for less than one hour, and no one was unable to sit comfortably on chair for 30 minutes.

These results are similar to the work done by [12].

\section{Conclusion}

It was found that the greater motion at the inner bearing correlated with better clinical results. Therefore the amount of motion at the two interfaces appears dependent on a number of variables and still a topic of debate.

\section{References}

[1] J. E.Gjertsen. The Norwegian Hip Fracture Register: experiences after the first 2 years and 15,576 reported operations," Acta Orthop, Vol.79(5), PP.583-593, 2008.

[2] M.Bhandari .Operative management of displaced femoral neck fractures in elderly patients: an international survey," JBJS, Vol. 87(9), PP.21222130, 2005.

[3] R. Iorio.Orthopaedic surgeon workforce and volume assessment for total hip and knee replacement in the United States: preparing for an epidemic," JBJS, vol. 90 (7), PP.1598-1605, 2008. 
[4] J. Tidermark. Quality of life and femoral neck fractures," Acta Orthop. Scand, Vol. 74(2), PP.162, 2003.

[5] J.F.Keating, A.Grant, M. Masson, N.W.Scott. Randomized comparison of reduction and fixation, bipolar hemiarthroplasty, and total hip arthroplasty: treatment of displaced intracapsular hip fractures in healthy older patients," JBJS, Vol. 88(2), PP.249-260, 2006.

[6] J.Tidermark, N.Zethraeus, O.Svensson. Femoral neck fractures in the elderly: functional outcome and quality of life according to EuroQol," Qual. life Res, Vol. 11(5), PP.473-481, 2002.

[7] M.P.J.van den Bekerom, I.N.Sierevelt, H.Bonke. The natural history of the hemiarthroplasty for displaced intracapsular femoral neck fractures: 302 patients followed until revision or death," Acta Orthop, Vol. 84(6), PP.555-560, 2013.

[8] G.S.Lausten, P.Vedel, P.-M. Nielsen. Fractures of the femoral neck treated with a bipolar endoprosthesis.," Clin. Orthop. Relat. Res., Vol.218, PP.63-67, 1987.

[9] M. Abdelkhalek, M. Abdelwahab, A.M. Ali. Bipolar versus fixed-head hip arthroplasty for femoral neck fractures in elderly patients," Strateg. Trauma Limb Reconstr., vol. 6(1), PP.16, 2011.

[10] P.von Roth, M. P. Abdel, W. S. Harmsen. Cemented bipolar hemiarthroplasty provides definitive treatment for femoral neck fractures at
20 years and beyond," Clin. Orthop. Relat. Res, Vol.473(11), PP.3595-3599, 2015.

[11] G.J.Haidukewych, T.A.Israel, D.J.Berry. Long-term survivorship of cemented bipolar hemiarthroplasty for fracture of the femoral neck," Clin. Orthop. Relat. Res., vol. 403, PP.118-126, 2002.

[12] S.Mazen, G.Julien, F.Riad. Retrospective evaluation of bipolar hip arthroplasty in fractures of the proximal femur," N. Am. J. Med. Sci., vol. 2(9), p. 409, 2010

[13] J.E.Kenzora . Outcome after hemiarthroplasty for femoral neck fractures in the elderly," Clin. Orthop. Relat. Res, Vol.348, PP.51-58, 1998.

[14] A.V.Bangarshettar .Intracapsular fracture neck of femur in elderly treated by hemiarthroplasty-a clinical study." RGUHS, Vol. 1(2), PP.12-28, 2006.

[15] S.Dixon , G.Bannister. Cemented bipolar hemiarthroplasty for displaced intracapsular fracture in the mobile active elderly patient," Injury, Vol. 35(2), PP.152-156, 2004.

[16] N.R.Lestrange. Bipolar arthroplasty for 496 hip fractures," Clin. Orthop. Relat. Res, Vol. 251, PP.7-19, 1990.

[17] P.Gallinaro, G.Tabasso, R.Negretto. Experience with bipolar prosthesis in femoral neck fractures in the elderly and debilitated.," Clin. Orthop. Relat. Res, Vol.251, PP.26-30, 1990. 